\title{
NASAL POLYPOSIS AND FUNGAL SCHIZOPHYLLUM COMMUNE INFECTION: A CASE REPORT
}

\author{
Aleksandar Perić1, Danilo Vojvodic $c^{2}$, Lidija Zolotarevski ${ }^{3}$, Aneta Peric ${ }^{4}$ \\ Military Medical Academy, Belgrade, Serbia: Clinic of Otorhinolaryngology, Department of Rhinology ${ }^{1}$, Institute of \\ Medical Research, Department of Clinical Immunology ${ }^{2}$, Institute of Pathology ${ }^{3}$, Institute of Pharmacy ${ }^{4}$

\begin{abstract}
Summary: We present a rare case of eosinophilic fungal rhinosinusitis with nasal polyps in a 32-year-old woman caused by basidiomycete fungus Schizophyllum commune. Diagnosis was done by the endoscopic nasal examination, computed tomography (CT) of the paranasal sinuses, the histopathological examination of polyps, the presence of eosinophils and fungal hyphae in nasal mucus and by the detection of $S$. commune by culture. The patient was successfully treated by combination of oral itraconazole and topical corticosteroid therapy combined with surgery. The pathogenesis and diagnosis of allergic fungal rhinosinusitis are also discussed.
\end{abstract}

Key words: Nasal polyposis; Allergic fungal rhinosinusitis; Schizophyllum commune; Endoscopic sinus surgery; Antifungal therapy; Itraconazole

\section{Introduction}

Chronic rhinosinusitis (CRS) is an inflammatory disease of the nose and paranasal sinuses that is present for at least 12 weeks without complete resolution (11). Nasal polyposis (NP), which is considered to be a subgroup of CRS, is defined as a chronic inflammatory disease of nasal and paranasal sinuses mucosa leading to diffuse formation of benign polyps protruding from sinuses into the nasal cavity (11). Although bacteria have long been implicated as pathogens in most forms of CRS and NP, it has been recognized that fungi may be responsible for some forms of CRS (9). Fungi are ubiquitous saprophytes that are reproduced by the formation of spores and can enter the respiratory tract by means of inhalation. They are continuously inhaled and deposited on the airway mucosa (15). Many fungi have been associated with sinus disease, including Aspergillus, Cladosporium, Alternaria, Penicillium and Mucor $(1,14,15)$.

Fungal sinusitis can be divided into two main types: invasive and non-invasive $(14,19)$. Invasive fungal sinusitis commonly occurs in patients with diabetes, or in individuals who are immunocompromised and is characterized by its invasiveness, tissue destruction and rapid onset. It spreads rapidly, via vascular invasion, into the orbit and central nervous system $(14,19)$. Non-invasive sinusitis can be divided into two forms: allergic fungal rhinosinusitis (AFRS), and sinus mycetoma (fungal ball), which occurs in immunocompetent patients $(14,19)$. AFRS should be suspected in individuals with intractable CRS, allergy and recurrent NP $(9,14,19)$. Ponikau et al. (15) suggested the term eosi- nophilic fungal rhinosinusitis because of striking role of the eosinophils in this disease which are probably triggered by the extramucosal fungi. In a study performed by Aydil et al. (2) fungal colonization was detected in $50 \%$ of the patients with massive NP. Erbek et al. (10) found a fungal allergy in $38.8 \%$ of allergic patients with NP.

Schizophyllum commune is a rare basidiomycetous fungus that has been reported as a cause of AFRS, invasive type of fungal sinusitis and allergic bronchopulmonary mycosis (ABPM) (4). We present a rare case of a woman with AFRS complicated by nasal polyps, caused by $S$. commune.

\section{Case report}

A 32-year-old woman presented with a one-year history of bilateral nasal obstruction, anosmia, bilateral purulent nasal discharge and facial pain. She had no history of systemic predispositions such as diabetes mellitus, neutropenia, immunodeficiency or previous corticosteroid therapy. Allergy to house-dust mite was determined by skin prick test. On the anterior rhinoscopy and endoscopic examination, we found bilateral nasal polyps in mucopurulent secretions that filled the nasal cavities. Computed tomography (CT) scan showed bilateral opacification of ethmoidal, maxillary and sphenoidal sinuses, soft tissue mass extending into the nasal cavities and bilateral mucosal thickening of the ostiomeatal complexes. There was no evidence of bone destruction. Peripheral blood test showed an increased number of eosinophils (11.5\%) and high levels of serum IgE (670 $\mathrm{IU} / \mathrm{ml})$. The patient started to use corticosteroid mometa- 


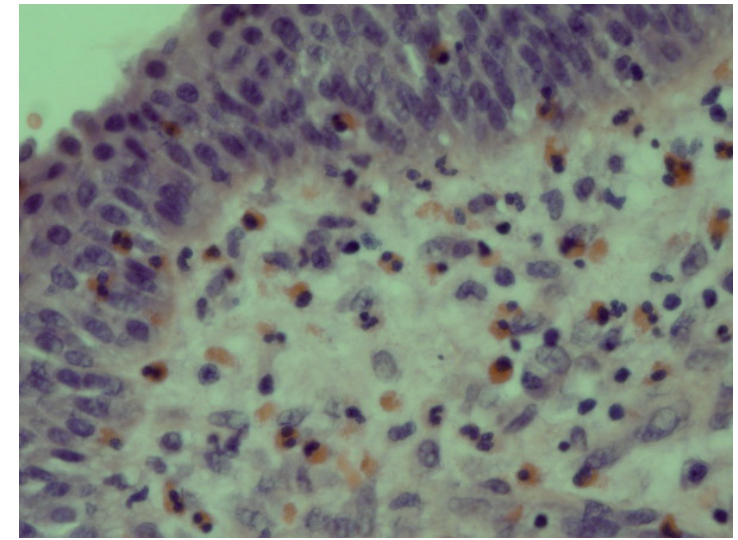

Fig. 1: Subepithelial and epithelial eosinophilic and lymphocytic infiltration in nasal polyp tissue (Haematoxylin \& Eosin, x 400 magnification).

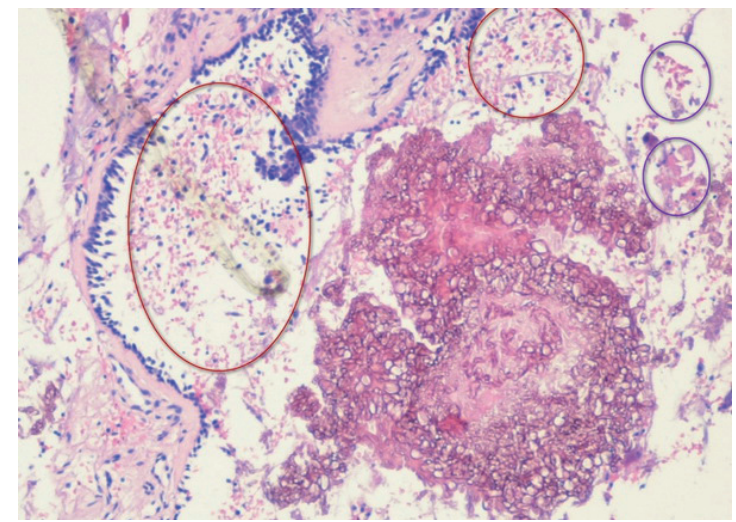

Fig. 2: Respiratory epithelium of the nasal polyp and fungal hyphae within the „allergic“ mucus. Typical sheets of degenerating eosinophils (red elipses) and Charcot-Leyden's crystals (blue elipses) are present (Haematoxylin \& Eosin, x 100 magnification).

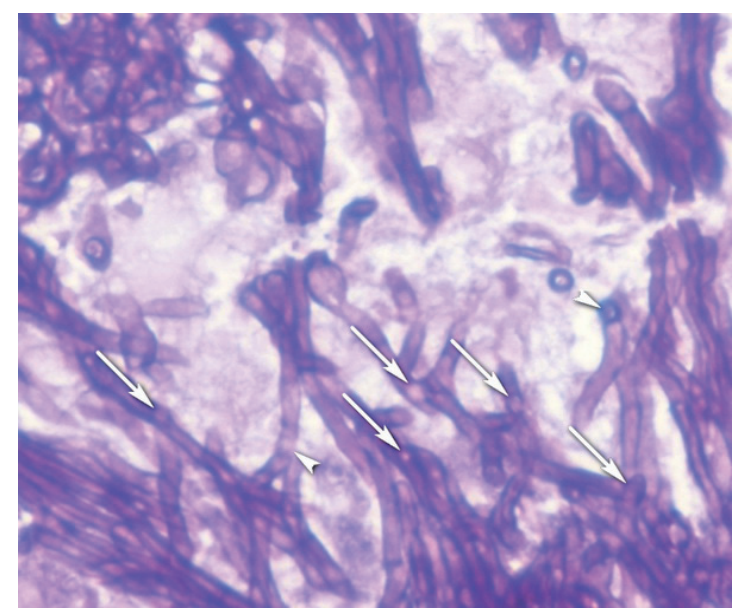

Fig. 3: The presence of fungal hyphae with typical clamp connections (arrow heads) (PAS, x 400 magnification). sone furoate intranasal spray ( $200 \mu \mathrm{g}$ daily, i.e., two $50 \mu \mathrm{g}$ sprays in each nostril in the morning) and macrolide antibiotic clarithromycin (1x500 mg daily) for two months. After that, there was no improvement.

The patient underwent bilateral endoscopic transnasal polypectomy, anterior and posterior ethmoidectomy, sphenoidotomy and bilateral middle meatal antrostomy. Polyps and inspissated mucus were removed from the sinuses and sent for culture and histological examination. Histology of the nasal polyps showed inflammed oedematous respiratory mucosa and extensive subepithelial eosinophilic and lymphocytic infiltrate (Fig. 1). In the mucus, we found numerous degenerated eosinophils and Charcot-Leyden's crystals (Fig. 2). The special staining for fungi was done. The Periodic Acid Schiff (PAS) technique showed the presence of hyphae with clamp connections (Fig. 3). The Gomori methenamine silver staining demonstrated scanty fungal hyphae scattered throughout the mucus. Cultures on Sabouraud's agar yielded a growth of a white mould. The older hyphae demonstrated the minute, peg-like outgrowths of wall material described on hyphae of $S$. commune. Bacterial cultures were positive only for saprophytic microbiota of coagulase-negative staphylococci. We concluded that the patient was suffering from AFRS. As the nasal symptoms did not improve two months following the surgery, we administrated oral antifungal therapy with itraconazole (200 $\mathrm{mg}$ daily) and topical mometasone furoate (200 $\mu \mathrm{g}$ daily) for two months. After the treatment, all nasal symptoms improved significantly. On the endoscopic examination, we found significantly smaller mucosal oedema. Four months after administered therapy, the polyps and purulent nasal discharge were not found. The patient has been followed up on an outpatient department for one year and has shown no signs of recurrence.

\section{Discussion}

The incidence of AFRS in cases of CRS treated surgically has been aproximately $6 \%$ to $10 \%(11,15)$. The diagnostic criteria of AFRS include endoscopic finding of nasal polyps, CRS (confirmed by CT scan), the presence of allergic mucin with degenerated eosinophils and the presence of fungi within this mucin (15). Six steps were found in the pathogenesis of AFRS (12). The host becomes sensitized to fungal antigens; fungal spores become trapped in nasal and sinus mucus and germinate into viable hyphae; in allergic rhinitis, the profile of T-cell cytokines in nasal tissue fits the class Th2 profile, with the production of interleukin (IL)-3, IL-4, IL-5, IL-13, regulated on activation, normal $\mathrm{T}$ cell expressed and secreted (RANTES), and granulocyte-macrophage colony-stimulating factor (GM-CSF); these cytokines give rise to IgE production with local mast cell degranulation and accumulation of eosinophils and allergen-specific Th2 cells in nasal tissue; eosinophils attack fungal hyphae and degranulate; the eosinophilic inflammatory process released multiple cytokines and growth fac- 
tors, wich may contribute to airway remodeling and nasal polyp formation (12). The local inflammatory response probably also triggers a systemic response with release of eosinophils from the bone marrow (12).

Although $S$. commune is ubiquitous basidiomycous fungus in nature, there were only rare reports of its association with human infections, including those of brain, lung and mouth $(6,13)$. Only 25 cases of sinusitis caused by $S$. commune have been reported yet $(1,3,6,7,8,17,18)$. Clark et al. (8) described the first case of AFRS associated with $S$. commune. Histological diagnosis was made on the mucinous material from the sinus which contained eosinophils, fungal hyphae and Charcot-Leyden's crystals. As well as in our patient, the fungal isolate was identified as $S$. commune on the basis of its morphology and minute peg-like outgrowths from vegetative hyphae and clamp connections. Ponikau et al. (15) suggested that the presence of CharcotLeyden's crystals as products of degenerating eosinophils is common in other diseases with eosinophil involvement. The presence of crystals is not specific for AFRS and it should not be used as a diagnostic criterion (15).

Invasive infections by basidiomycete fungi are extremely rare. Rosenthal et al. (17) reported such an infection in a HIV-positive patient who presented with maxillary sinusitis associated with the $S$. commune infection. Sigler et al. (18) described a case of maxillary sinusitis in a diabetic woman caused by this fungus.

In our case, diagnosis of AFRS was based on the endoscopic and CT findings, the presence of eosinophils and fungal hyphae in nasal secretions and on the histopathologically confirmed tissue eosinophilia. We isolated S. commu$n e$ by culture. $S$. commune infection may be misdiagnosed because presentation of infection and histopathological findings can be suggestive of sinusitis caused by Aspergillus species. The hyphae of $S$. commune may appear similar to those of Aspergillus. Identification of the isolate was done by its typical features. Subcultures formed sterile medusoid structures from nonclamped mycelia until spontaneous dikaryotization resulted in the development of characteristic fan-shaped fruiting bodies. Identification was confirmed by the presence of spicules formed on the hyphae and by vegetative compatibility with known isolates. The mycological diagnosis of $S$. commune is easy to make if hyphae bearing spicules or clamp connections are present. Identification is especially difficult in the case of monokaryotic isolates, which, unlike dikaryotic ones, are devoid of clamp connections. The case presented by Baron et al. (3) indicated that molecular diagnostics by nucleotide sequencing is very helpful to accurately identify this fungus, especially when mycological features are atypical.

The patient was successfully treated by combination of antifungal/ corticosteroid and surgical therapy. Previous studies showed that corticosteroid therapy and surgery were main therapy strategy in AFRS $(9,11,19)$. Systemic antifungal drugs such as amphotericin B, voriconazole or caspofungin were not effective (4). Although preliminary trials suggest a beneficial effect of topical (amphotericin B) and oral (terbinafine) antifungal agents, several doubleblind placebo controlled trials do not $(5,9,11)$. In experience of our hospital, the results of itraconazole treatment with a prolonged follow-up seem good for AFRS caused by Aspergillus. In our case, which was the first case of rhinosinusitis caused by $S$. commune reported from our institution, we administrated oral itraconazole and topical mometasone furoate in treatment of $S$. commune infection and eosinophilic inflammation which was beneficial by giving significant improvement in combination with endoscopic sinus surgery. Only one case of a similarly treated patient with AFRS associated with $S$. commune infection has been previously reported from Japan (1). In the case of pulmonary $S$. commune presented by Ishiguro et al. (13), the symptoms of disease improved with itraconazole administration without steroid therapy, which may indicate that the infectious aspect was more likely than the allergic aspect in pathogenesis of this disease. It is possible that itraconazole reduced $S$. commune, an allergic antigen, which resulted in attenuated allergic reaction. However, our experiences showed that topical corticosteroids are necessary in treatment of AFRS, because of their antiinflammatory and antioedematous effects.

\section{Conclusion}

This case report expands the list of unusual infections associted with CRS and NP. Our report showed that NP can sometimes be associated with fungal $S$. commune infection. The participation of rhinologist, pathologist, and microbiologist is necessary in diagnosis of such rare forms of rhinosinusitis. Surgical treatment alone is not successful and it should be combined with antifungal and topical corticosteroid treatment.

\section{Acknowledgements}

We would like to thank dear colleagues from Institute of Microbiology, Military Medical Academy, Belgrade, Serbia for their support in diagnosis of this rare form of AFRS.

\section{References}

1. Ahmed MK, Ishino T, Takeno S, Hirakawa K. Bilateral allergic fungal rhinosinusitis caused by Schizophyllum commune and Aspergillus niger. Rhinology 2009;47: 217-21.

2. Aydil U, Kalkanci A, Ceylan A, Berk E, Kustimur S, Uslu S. Investigation of fungi in massive nasal polyps: microscopy, culture, polymerase-chain reaction, and serology. Am J Rhinol 2007;21:417-22.

3. Baron O, Cassang S, Percodani J, et al. Nucleotide sequecing for diagnosis of sinusal infection by Schizophyllum commune, an uncommon pathogenic mucus. J Clin Microbiol 2006;44:3042-3.

4. Braun H, Buzina W, Freudenschuss K, Beham A, Stammberger H. „Eosinophilic fungal rhinosinusitis“" a common disorder in Europe? Laryngoscope 2003;113:264-9.

5. Braun JJ, Pauli G, Schultz P, Gentine A, Ebbo D, de Blay F. Allergic fungal sinusitis associated with allergic bronchopulmonary aspergillosis: an uncommon sinobronchial allergic mycosis. Am J Rhinol 2007; 21:412-6.

6. Buzina W, Braun H, Freudenschuss K, Lacker A, Schimpl K, Stammberger H. The basidiomycete Schizophyllum commune in paranasal sinuses. Mycoses 2003; 46 Suppl 1:23-7.

7. Castro LA, Alvarez MI, Martinez E. Case report of Schizophyllum commune sinusitis in an immunocompetent patient. Colomb Med 2010;41:71-5. 
8. Clark S, Campbell CK, Sandison A, Choa DI. Schizophyllum commune: an unusual isolate from a patient with allergic fungal sinusitis. J Infect 1996; 32:147-50.

9. Ebbens, FA, Georgalas C, Rinia AB, van Drunen CM, Lund VJ, Fokkens WJ. The fungal debate: where do we stand today? Rhinology 2007;45:178-89.

10. Erbek SS, Topal Ö, Erbek S, Cakmak Ö. Fungal allergy in chronic rhinosinusitis with or without polyps. Kulak Burun Bogaz Ihtis Derg 2008;18:153-6.

11. Fokkens WJ, Lund VJ, Mullol J; European Position Paper on Rhinosinusitis and Nasal Polyps Group. EP3OS 2007: European position paper on rhinosinusitis and nasal polyps 2007. A summary for otorhinolaryngologist. Rhinology 2007;45:97-101.

12. Hamilos DL, Lund VJ. Etiology of chronic rhinosinusitis: the role of fungus. Ann Otol Rhinol Laryngol Suppl 2004;193:27-31.

13. Ishiguro T, Takayanagi N, Tokunaga D, et al. Pulmonary Schizophyllum commune infection developing mucoid impaction of the bronchi. Yale J Biol Med 2007; 80:105-11.
14. Michael RC, Michael JS, Ashbee RH, Mathews MS. Mycological profile of fungal sinusitis: An audit of specimens over a 7-year period in a tertiary care hospital in Tamil Nadu. Indian J Pathol Microbiol 2008;51:493-6.

15. Ponikau JU, Sherris DA, Kern EB, Homburger HA, Frigas E, Gaffey TA, et al. The diagnosis and incidence of allergic fungal sinusitis. Mayo Clin Proc 1999;74:877-84.

16. Ragab A, Clement P, Vincken W, Nolard N, Simones F. Fungal cultures in different parts of the upper and lower airways in chronic rhinosinusitis. Rhinology 2006;44: 19-25.

17. Rosenthal J, Katz R, DuBois DB, Morrissey A, Machicao A. Chronic maxillary sinusitis associated with the mushroom Schizophyllum commune in a patient with AIDS. Clin Infect Dis 1992;14:46-8.

18. Sigler L, Bartley JR, Parr DH, Morris AJ. Maxillary sinusitis caused by medusoid form of Schizophyllum commune. J Clin Microbiol 1999;37:3395-8.

19. Trasher RD, Kingdom TT. Fungal infections of the head and neck: an update. Otolaryngol Clin North Am 2003;36:577-94.

Received: $24 / 11 / 2010$.

Accepted in revised form: 29/12/2010.

\section{Corresponding author:}

Dr. Aleksandar Perić, Clinic of Otorhinolaryngology, Department of Rhinology, Military Medical Academy, Crnotravska 17, 11040 Belgrade, Serbia; e-mail alexneta@sezampro.rs 